\title{
NGC 3894: a young radio galaxy seen by Fermi-LAT
}

\author{
G. Principe ${ }^{1}$, G. Migliori ${ }^{1,2}$, T. J. Johnson ${ }^{3}$, F. D’ Ammando ${ }^{1}$, M. Giroletti ${ }^{1}$, M. Orienti ${ }^{1}$, C. Stanghellini ${ }^{1}$, \\ G. B. Taylor ${ }^{4}$, E. Torresi ${ }^{5}$, and C. C. Cheung ${ }^{6}$ \\ 1 INAF - Istituto di Radioastronomia, Bologna, Italy \\ e-mail: giacomo.principe@inaf.it \\ 2 Dip. di Fisica e Astronomia, Università di Bologna, Bologna, Italy \\ 3 College of Science, George Mason University, Resident at Naval Research Laboratory, Washington DC, USA \\ ${ }^{4}$ University of New Mexico, Albuquerque, NM, USA \\ 5 INAF - Osservatorio di Astrofisica e Scienza dello Spazio di Bologna, Bologna, Italy \\ 6 Naval Research Laboratory, Space Science Division, Washington DC, USA \\ Received 4 November 2019 / Accepted 28 February 2020
}

\begin{abstract}
Context. According to radiative models, radio galaxies may produce $\gamma$-ray emission from the first stages of their evolution. However, very few such galaxies have been detected by the Fermi Large Area Telescope (LAT) so far.

Aims. NGC 3894 is a nearby $(z=0.0108)$ object that belongs to the class of compact symmetric objects (CSOs, i.e., the most compact and youngest radio galaxies), which is associated with a $\gamma$-ray counterpart in the Fourth Fermi-LAT source catalog. Here we present a study of the source in the $\gamma$-ray and radio bands aimed at investigating its high-energy emission and assess its young nature.

Methods. We analyzed 10.8 years of Fermi-LAT data between $100 \mathrm{MeV}$ and $300 \mathrm{GeV}$ and determined the spectral and variability characteristics of the source. Multi-epoch very long baseline array (VLBA) observations between 5 and $15 \mathrm{GHz}$ over a period of 35 years were used to study the radio morphology of NGC 3894 and its evolution.

Results. NGC 3894 is detected in $\gamma$-rays with a significance $>9 \sigma$ over the full period, and no significant variability has been observed in the $\gamma$-ray flux on a yearly time-scale. The spectrum is modeled with a flat power law $(\Gamma=2.0 \pm 0.1)$ and a flux on the order of $2.2 \times 10^{-9} \mathrm{ph} \mathrm{cm}^{-2} \mathrm{~s}^{-1}$. For the first time, the VLBA data allow us to constrain with high precision the apparent velocity of the jet and counter-jet side to be $\beta_{\text {app, NW }}=0.132 \pm 0.004$ and $\beta_{\text {app,SE }}=0.065 \pm 0.003$, respectively.

Conclusions. Fermi-LAT and VLBA results favor the youth scenario for the inner structure of this object, with an estimated dynamical age of $59 \pm 5$ years. The estimated range of viewing angle $\left(10^{\circ}<\theta<21^{\circ}\right)$ does not exclude a possible jet-like origin of the $\gamma$-ray emission.
\end{abstract}

Key words. Galaxy: evolution - galaxies: nuclei - galaxies: general - galaxies: jets - radio continuum: galaxies gamma-rays: galaxies

\section{Introduction}

The extragalactic $\gamma$-ray sky is dominated by blazars (Massaro et al. 2015), for which the $\gamma$-ray emission is favored by the small jet inclination angle and beaming effect. However, the increasing amount of data collected by the Large Area Telescope (LAT) on board the Fermi Gamma-ray Space Telescope (Atwood et al. 2009) allows us to also investigate other classes of objects in the $\gamma$-ray sky (Sahakyan et al. 2018). A small percentage, $2 \%$ in the fourth catalog of $\gamma$-ray AGN (4LAC, The Fermi-LAT Collaboration 2019), is represented by radio galaxies (or misaligned AGN), which have larger jet inclination angles $\left(>10^{\circ}\right)$ and a smaller Doppler factor $(\delta \leq 2-3)$.

Compact symmetric objects (CSOs, i.e., the most compact and youngest radio galaxies), with their symmetric and subkiloparsec radio structure, are important objects because they are expected to be the progenitors of the extended radio galaxies (Readhead et al. 1996). In principle, CSOs with a powerful jet could produce $\gamma$-ray emission up to the $\mathrm{GeV}$ band through inverse Compton scattering of the ultraviolet (UV) photons from the accretion disk by the electrons in the compact radio lobes (Stawarz et al. 2008; Kino \& Asano 2011, see the second reference for a discussion of hadronic models). However, systematic searches of CSOs in $\gamma$-rays have so far been rather unsuccessful
(D'Ammando et al. 2016). Dedicated studies have reported a handful of detections of CSO candidates (McConville et al. 2011; Müller et al. 2014). Of these, the nearby $(z=0.0144)$ radio galaxy NGC 6328 (also called PKS 1718-649) is the most probable case (Migliori et al. 2016), which has recently been confirmed in the Fourth Fermi-LAT Source Catalog (4FGL, The Fermi-LAT Collaboration 2020).

The 4FGL catalog reports the association of a newly detected $\gamma$-ray source (4FGL J1149.0+5924) with the radio galaxy NGC 3894 (also known as UGC 06779 or $1146+596$ ), which is also classified as a CSO (Peck \& Taylor 2000). NGC 3894 $(z=0.010751 \text {, de Vaucouleurs et al. 1991) })^{1}$ is one of the least radio-luminous CSOs; it is hosted in an elliptical galaxy whose optical continuum emission is dominated by starlight (Perlman et al. 2001). On the basis of the $[\mathrm{OIII}] / \mathrm{H} \beta$ versus $[\mathrm{NII}] / \mathrm{H} \alpha$ and $[\mathrm{OI}] / \mathrm{H} \alpha$, Gonçalves \& Serote Roos (2004) classified the source as a low-ionization emission-line region (LINER), possibly indicating a low-power central engine. Observations with very long baseline interferometry (VLBI) over 15 years allowed the identification of the radio core and provided evidence for twin parsecscale jets (Peck \& Taylor 1998). Both the jets were estimated to

1 At this luminosity distance $(50.1 \mathrm{Mpc}), 1$ mas corresponds to $0.23 \mathrm{pc}$. 
be mildly relativistic $(v \sim 0.3 c)$ and oriented away from the line of sight $\left(\theta \sim 50^{\circ}\right.$, Taylor et al. 1998).

In this paper, we investigate the $\gamma$-ray and radio properties of NGC 3894. We analyze 10.8 years of Fermi-LAT data between $100 \mathrm{MeV}$ and $300 \mathrm{GeV}$ as well as several epochs of VLBA radio data, extending the Taylor et al. (1998) coverage from 15 years to $\sim 35$ years, in order to investigate the high-energy characteristics of NGC 3894 and verify its young nature.

Throughout this article, we assume $H_{0}=70 \mathrm{~km} \mathrm{~s}^{-1} \mathrm{Mpc}^{-1}$, $\Omega_{\mathrm{M}}=0.3$, and $\Omega_{\Lambda}=0.7$ in a flat Universe.

\section{Fermi data and analysis}

The LAT is a $\gamma$-ray telescope that detects photons by conversion into electron-positron pairs. It has an operational energy range of $20 \mathrm{MeV}-300 \mathrm{GeV}$ and beyond. The LAT is comprised of a high-resolution converter tracker (for direction measurement of the incident $\gamma$-rays), a $\operatorname{CsI}(\mathrm{Tl})$ crystal calorimeter (for energy measurement), and an anticoincidence detector to identify the background of charged particles (Atwood et al. 2009).

We performed a dedicated analysis of the Fermi-LAT data with the goals of (1) confirming the detection and spatial association of 4FGL 1149.0+5924 with the CSO NGC 3894, (2) determining its $\gamma$-ray spectral properties, and (3) investigating its temporal behavior. We collected $\sim 10.8$ years of Pass 8 LAT data (Atwood et al. 2013) that were collected between August 4, 2008, and June 20, 2019, which means that we consider a more extended dataset than in the 4FGL (8 years). We selected events that were reprocessed with the P8R3_Source_V2 instrument response functions (IRFs; Bruel et al. 2018) in the energy range between $100 \mathrm{MeV}$ and $300 \mathrm{GeV}$ because below $100 \mathrm{MeV}$ the source is not detected (Principe et al. 2018). The region of interest (ROI) has a radius of about $20^{\circ}$ that is centered on the position of the $\gamma$-ray source 4FGL J1149.0+5924, as reported in the 4FGL catalog $($ RA, Dec $(J 2000)=177.25,59 \circ 42$, The FermiLAT Collaboration 2020).

The binned likelihood analysis (which consists of model optimization, localization, and a study of the spectrum and variability) was performed with Fermipy ${ }^{2}$ (Wood et al. 2017), a python package that facilitates analysis of data from the LAT with the Fermi science tools, of which the version 11-07-00 was used. The maps were created with a pixel size of $0.1^{\circ}$.

$\gamma$-rays with a zenith angle larger than $90^{\circ}$, as well as photons below $300 \mathrm{MeV}$ from the point spread function (PSF) 0 event type $^{3}$, were excluded in order to eliminate most of the contamination from secondary $\gamma$-rays from the Earth's limb (Abdo et al. 2009). The model used to describe the sky includes all point-like and extended LAT sources that are located at a distance $<25^{\circ}$ from the source position, as listed in the 4FGL, as well as the Galactic diffuse and isotropic emission. For these two latter contributions, we made use of the same templates $^{4}$ as were adopted to compile the 4FGL. For the analysis we first optimized the model for the ROI (fermipy.optimize), then we searched for the possible presence of new sources (fermipy.find_sources), and finally, we relocalized the source (fermipy.localize). We investigated the possible presence of additional faint sources that are not in 4FGL by generating test

\footnotetext{
2 http://fermipy.readthedocs.io/en/latest/

3 A measure of the quality of the direction reconstruction is used to assign events to four quartiles. $\gamma$-rays in Pass 8 data can be separated into four PSF event types: 0, 1, 2, and 3, where PSF0 has the largest PSF and PSF3 has the best.

4 https://fermi.gsfc.nasa.gov/ssc/data/access/lat/ BackgroundModels.html
}

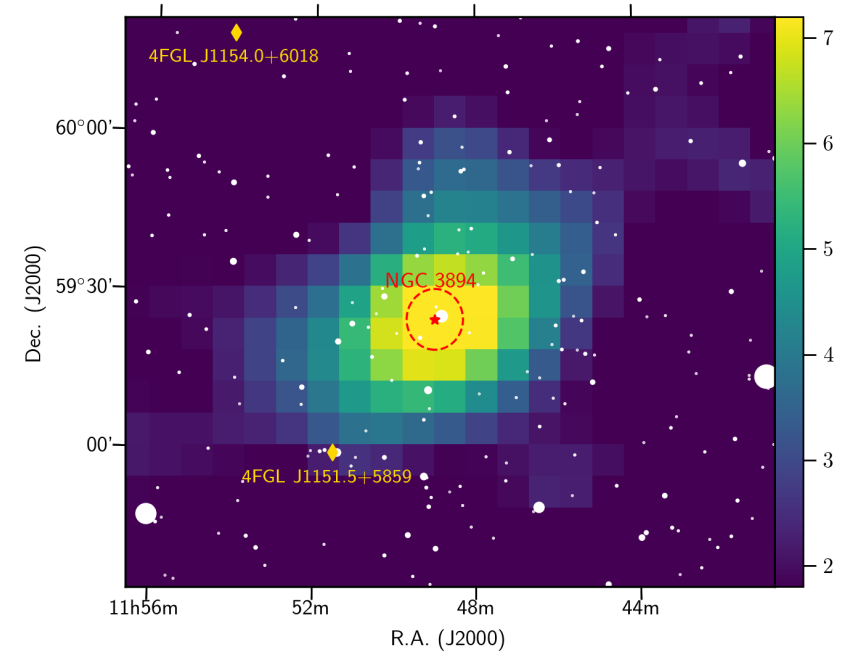

Fig. 1. Fermi-LAT TS map (in sigma units) above $3 \mathrm{GeV}$, the red star and dashed circle represent the central position and the $95 \%$ confidencelevel uncertainty $R_{95}=0.05^{\circ}$ of the $\gamma$-ray source, respectively. White dots show radio sources from the NVSS survey scaled depending on their flux.

statistic (TS) maps. The test statistic is the logarithmic ratio of the likelihood of a model with the source being at a given position in a grid to the likelihood of the model without the source, $\mathrm{TS}=2 \log \left(\frac{\text { likelihood }}{\text { likel }}\right)$ (Mattoox et al. 1996). We found three new sources that we added to our model. The best-fit positions of these new sources are RA, Dec $(J 2000)=\left(182.62^{\circ}, 59.15^{\circ}\right)$, $\left(180.96^{\circ}, 58.31^{\circ}\right)$ and $\left(177.36^{\circ}, 62.77^{\circ}\right)$, with a $95 \%$ confidencelevel uncertainty $R_{95}=0.07^{\circ}$.

We left free to vary the diffuse backgrounds and the spectral parameters of the sources within $5^{\circ}$ of our target. For the sources in a radius between $5^{\circ}$ and $10^{\circ}$ only the normalization was fit, while we fixed the parameters of all the sources within the ROI at larger angular distances from our target. The spectral fit was performed over the energy range from $100 \mathrm{MeV}$ to $300 \mathrm{GeV}$.

\section{Fermi-LAT results on NGC 3894}

The results of the localization analysis present a significant excess at a TS $=98$, corresponding to a significance $>9 \sigma$, centered on the position $(\mathrm{RA}, \operatorname{Dec}(\mathrm{J} 2000))=\left(177.25^{\circ} \pm 0.02^{\circ}\right.$, $\left.59.41^{\circ} \pm 0.02^{\circ}\right), 95 \%$ confidence-level uncertainty $R_{95}=0.05^{\circ}$. This is compatible with the position of 4FGL J1149.0+5924.

NGC 3894, with an offset of $0.024^{\circ}$ and a flux density of $482 \mathrm{mJy}$, is the nearest bright radio source (in the NVSS survey) to 4FGL J1149.0+5924, making it the best candidate radio counterpart. No other NVSS sources are detected within the $R_{\mathrm{LAT}, 95}$ down to $4 \mathrm{mJy}$. NGC 3894 has also been proposed as the most likely counterpart in the 4LAC using both the Bayesian method (Probability $\sim 0.998$ ) based on spatial coincidence and the likelihood ratio method (Probability $\sim 0.968$ ) based on radio flux density. Figure 1 shows the Fermi-LAT TS map (in sigma units) above $3 \mathrm{GeV}$ for the region around NGC 3894.

\subsection{Fermi-LAT spectral energy distribution}

We modeled the spectrum of the source with a power-law function $\left(\frac{\mathrm{d} N}{\mathrm{~d} E}=N_{0} \times\left(\frac{E}{E_{\mathrm{b}}}\right)^{-\Gamma}\right)$. The best-fit results for the $\gamma$-ray source associated with NGC 3894 are $\Gamma=2.01 \pm 0.10$ and 


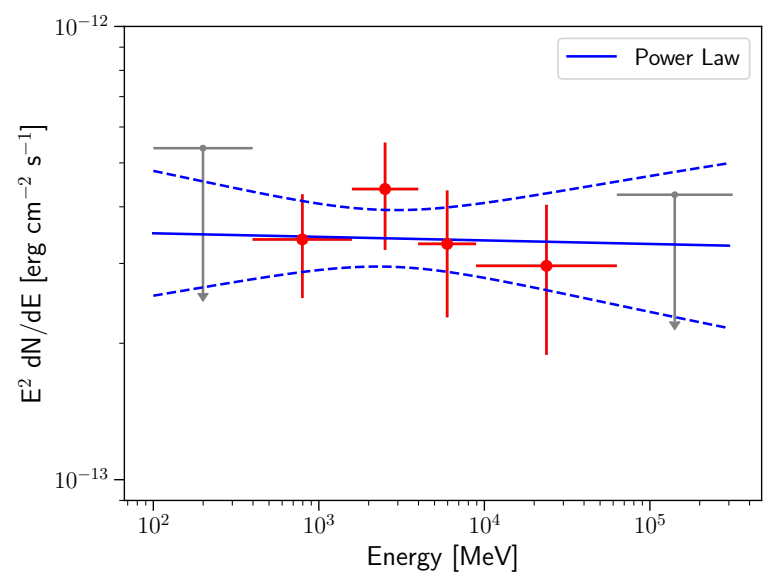

Fig. 2. Fermi-LAT spectrum of NGC 3894 obtained using 10.8 years of data between $100 \mathrm{MeV}$ and $300 \mathrm{GeV}$. The spectrum was fit with a power law (blue line). The $1 \sigma$ upper limit is reported when TS $<10$.

$N_{0}=(2.14 \pm 0.39) \times 10^{-13}\left(\mathrm{MeV} \mathrm{cm}^{-2} \mathrm{~s}^{-1}\right)$ for $E_{0}=1 \mathrm{GeV}$, which are in agreement with the 4FGL results for this source $\left(\Gamma_{4 \mathrm{FGL}}=2.06 \pm 0.12\right)$. Figure 2 shows the spectral energy distribution (SED) we obtained here using 10.8 years of Fermi-LAT.

\subsection{Fermi-LAT study of emission variability}

Over a period of eight years, the source presents a variability index of $\mathrm{TS}_{\mathrm{var}}=0.97^{5}$ in the 4FGL, where a value of $\mathrm{TS}_{\mathrm{var}}>18.5$ is used to identify a variable sources at $99 \%$ confidence level. In our analysis we extended the study of the $\gamma$-ray emission variability to a period of 10.8 years in order to investigate possible presence of high activity in the last three years. To perform a study of the $\gamma$-ray emission variability of NGC 3894, we divided the Fermi-LAT data into time intervals of one year; ten months was used for the last bin. For the light-curve analysis we fixed the photon index to the value obtained for 10.8 years of data, $\Gamma=2.01$ (see Sect. 3), and left only the normalization free to vary. The $95 \%$ upper limit was reported in each time interval with $\mathrm{TS}<10$. Figure 3 shows the light curve for time bins of about one year, using 10.8 years of Fermi-LAT data.

No indication of variability is observed in the $\gamma$-ray flux of NGC 3894: a test of the uniform distribution for the averaged flux $F=(2.2 \pm 1.0) \times 10^{-9} \mathrm{ph} \mathrm{cm}^{-2} \mathrm{~s}^{-1}$ returns a value of $\chi^{2} / \mathrm{ndf}=2.3 / 10$. Our results extend for a longer period what has been obtained in the 4FGL and confirm that there is no indication of $\gamma$-ray flux variability of NGC 3894, as seen for most misaligned sources.

\section{New radio results on NGC 3894}

To complement the datasets presented by Taylor et al. (1998), we downloaded VLBA archival data for five epochs of observations spanning 1995 to 2017 between 5 and $8 \mathrm{GHz}$. Table 1 presents the VLBA archival datasets we used.

We carried out amplitude and phase calibration in a standard way following the guidelines given in Appendix C of the Astronomical Image Processing System (AIPS) cook book (Greisen 2003). Data were imaged in AIPS after some iterations of phase self-calibration. Model fitting of the calibrated $(u, v)$-data ${ }^{6}$ was

\footnotetext{
5 A measure of the variability index.

6 The Fourier transform of the sky brightness distribution observed by interferometers.
}

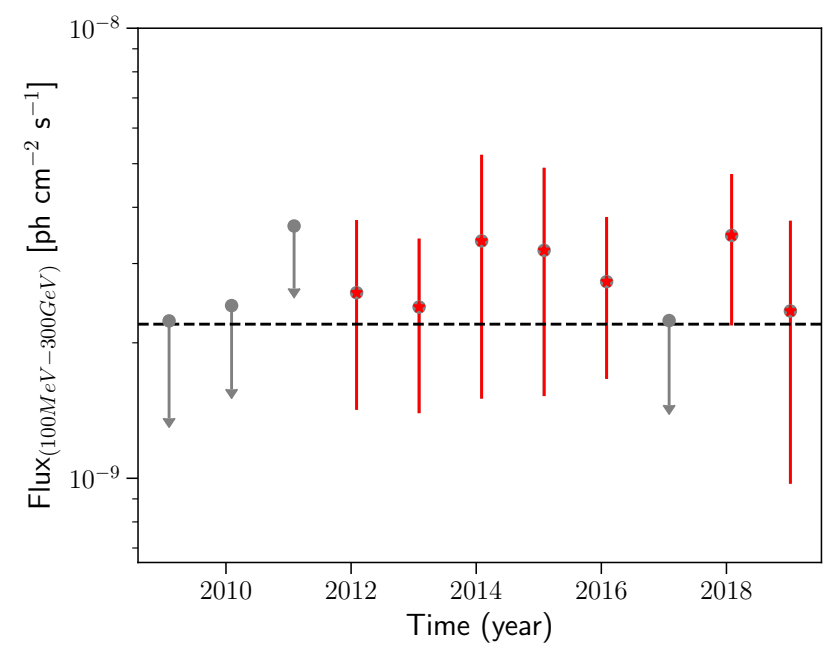

Fig. 3. Fermi-LAT one-year binned light curve of NGC 3894. The dashed line represents the averaged flux for the entire period Flux $_{10.8 \text { years }}=2.2 \times 10^{-9} \mathrm{ph} \mathrm{cm}^{-2} \mathrm{~s}^{-1}$.

Table 1. VLBA archive data.

\begin{tabular}{cccl}
\hline \hline Project & Date & $v(\mathrm{GHz})$ & Notes \\
\hline BR026 & 22 Mar. 1995 & 8.4 & NRAO \\
BV019 & 24 Aug. 1996 & 5 & NRAO \\
BT094 & 30 Jun. 2007 & 8.4 & NRAO \\
RV122 & 25 Apr. 2017 & 8.4 & Astrogeo \\
RV122 & 04 Aug. 2017 & 8.6 & Astrogeo \\
\hline
\end{tabular}

Notes. Column (4) lists NRAO data downloaded from the archive and calibrated as described in the text, and Astrogeo are calibrated visibility data downloaded from astrogeo. org and that were only analyzed with Modelfit in Difmap. The RV122 experiment is described in Petrov et al. (2009).

performed with the task MODELFIT available in the software package DIFMAP (Shepherd et al. 1994).

The radio source appears two-sided with respect to a central brightest flat-spectrum component identified as the radio core (see Fig. 4; for a spectral index map, see also Fig. 2I in Tremblay et al. 2016). The structure is resolved into different components that are elongated over $\sim 20$ mas at position angle $\sim-40^{\circ}$ (measured positive north-to-east), corresponding to an overall projected size of $\sim 4 \mathrm{pc}$. Some more distant and diffuse emission is recovered, particularly on the southern side, in the $5 \mathrm{GHz}$ datasets, in agreement with what has been reported at 1.4-1.6 GHz by Peck \& Taylor (1998) and Taylor et al. (1998). The data have rather different distributions of the visibilities, therefore a precise association and comparison of the distinct components seen at different epochs or frequencies is challenging. In our model-fit procedure, we used a minimal number of discrete Gaussian components.

The outermost NW and SE external components are consistently detected across epochs and frequencies. In Table 2 and Fig. 5 we show the separation of the NW and SE component from the central core as a function of time. We assume that the core is stationary across epochs and that any opacity-related shift in its position is negligible in comparison with the observational uncertainty on the position of each component. We only considered very close frequencies $(5$ and $8.4 \mathrm{GHz}$ ) and rather extended components, therefore this is a sensible assumption. 


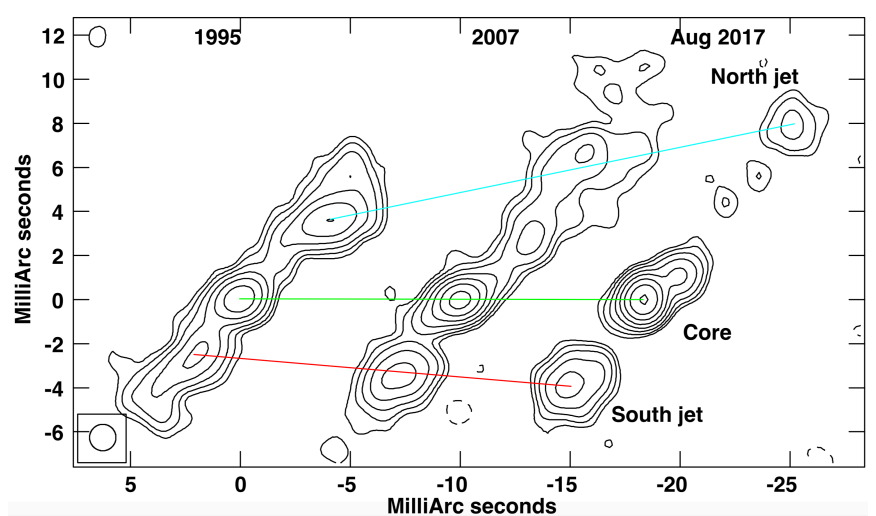

Fig. 4. Comparison of three VLBA epochs at $8.4 \mathrm{GHz}$ in 1995 , 2007, and August 2017 (left to right). Images are spaced horizontally by a distance proportional to the time separation. Contours are traced at $\pm(1,2,3,8,16, \ldots) \times 1.5 \mathrm{mJy}$ beam $^{-1}$. The colored lines trace the central position of the NW jet (blue), SE jet (red), and core (green), respectively. All the images are convolved with the same circular beam (shown in the bottom left corner), whose full width at half-maximum is 1.2 mas.

Table 2. Separation of NW and SE components with respect to the core.

\begin{tabular}{ccccccc}
\hline \hline Epoch & $\begin{array}{c}v \\
(\mathrm{GHz})\end{array}$ & $\begin{array}{c}r_{\mathrm{NW}} \\
(\mathrm{mas})\end{array}$ & $\begin{array}{c}\theta_{\mathrm{NW}} \\
\left({ }^{\circ}\right)\end{array}$ & $\begin{array}{c}r_{\mathrm{SE}} \\
(\mathrm{mas})\end{array}$ & $\begin{array}{c}\theta_{\mathrm{SE}} \\
\left({ }^{\circ}\right)\end{array}$ & Ref. \\
\hline 1981.9 & 5 & $2.5 \pm 2.0$ & -49.0 & $3.2 \pm 2.0$ & -225.0 & 1 \\
1989.3 & 5 & $4.2 \pm 1.0$ & -48.4 & $4.1 \pm 1.0$ & -221.3 & 2 \\
1992.2 & 5 & $5.0 \pm 0.3$ & -42.8 & $3.9 \pm 0.3$ & -223.1 & 2 \\
1994.7 & 5 & $5.1 \pm 0.3$ & -46.8 & $3.7 \pm 0.3$ & -219.1 & 2 \\
1995.2 & 8.4 & $5.6 \pm 0.2$ & -47.1 & $3.5 \pm 0.3$ & -218.0 & 3 \\
1996.6 & 5 & $6.0 \pm 0.3$ & -46.9 & $3.3 \pm 0.2$ & -217.1 & 3 \\
2007.5 & 8.4 & $8.2 \pm 0.6$ & -41.7 & $4.5 \pm 0.2$ & -219.0 & 3 \\
2017.3 & 8.4 & $10.2 \pm 0.6$ & -42.0 & $5.0 \pm 0.2$ & -221.4 & 3 \\
2017.6 & 8.6 & $10.2 \pm 0.3$ & -40.4 & $5.0 \pm 0.2$ & -220.1 & 3 \\
\hline
\end{tabular}

Notes. Uncertainties are derived from the component size along the direction to the core, divided by a factor 5 , except for the first two epochs: for 1981.9, in which the confusion is severe and the $(u, v)$ coverage sparse, we adopt an uncertainty of 2 mas, while for 1989.3 , which has better quality and somewhat larger separation, we adopt 1 mas.

References. 1: Wrobel et al. (1985), 2: Taylor et al. (1998), and 3: this work.

As was noted by Taylor et al. (1998), the source is not well described by simple Gaussian components, and caution should be used in interpreting the results, particularly for the SE side component. However, based on our now 35-year long time baseline, we can determine the motion on the NW side with high precision and use it as a prior for a self-consistent scenario describing the kinematics of NGC 3894. In particular, we successfully fit our data with a model in which the two components were both ejected at $t_{\mathrm{ej}}=1961 \pm 5$, corresponding to a dynamical age of $59 \pm 5$ years, and they are separating from each other at a rate of $(0.269 \pm 0.007)$ mas $\mathrm{yr}^{-1}$, or in units of the speed of light, $\beta_{\text {app }}=v_{\text {app }} / c=0.202 \pm 0.005$. In this scenario, the individual speeds of the two components are $\beta_{\mathrm{app}, \mathrm{NW}}=0.132 \pm 0.004$ and $\beta_{\text {app,SE }}=0.065 \pm 0.003$.

We can use these results and the well-known relation for the arm-length ratio

$D=\frac{\mu_{\mathrm{NW}}}{\mu_{\mathrm{SE}}}=\frac{d_{\mathrm{NW}}}{d_{\mathrm{SE}}}=\frac{1+\beta \cos \theta}{1-\beta \cos \theta}$

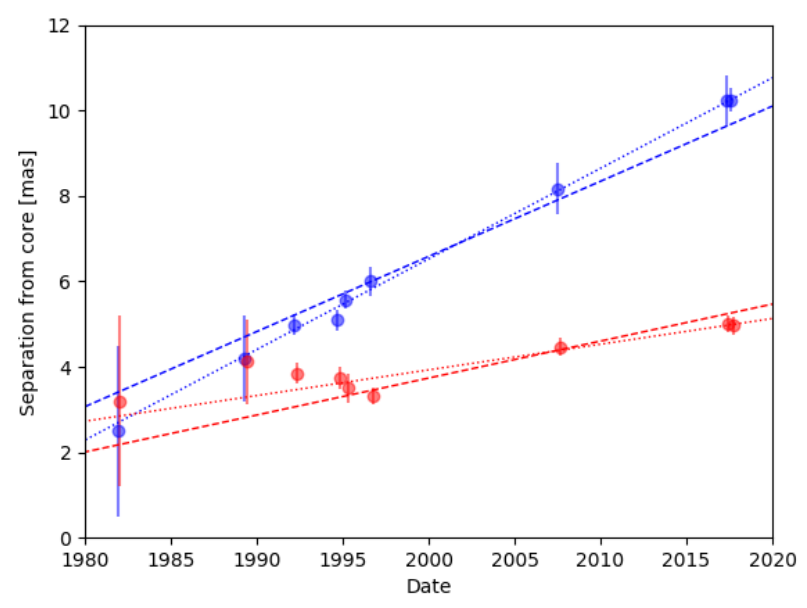

Fig. 5. Separation from core for the NW (in blue) and SE (in red) components. For each set of points (see also Table 2), the dotted line shows an unconstrained linear fit to the data, while the dashed line includes the additional condition that the ejection dates of the two components are consistent with each other.

where $\mu$ (mas/years) is the proper motion of each component (jet and counter jet) with respect to the core, to set a first constraint on $\beta \cos \theta \geq 0.34 \pm 0.03$, because our measurements yield $D=$ $2.03 \pm 0.12$.

The other complementary constraint that is usually provided by the brightness ratio is more ambiguous in our case because the flux density of each component varies from epoch to epoch, and their ratio does not have a constant value or even a welldefined trend. It is not clear how systematic effects due to the changes in $(u, v)$-coverage can be separated from more physical causes, which involve not only the values of $\beta$ and $\theta$, but also the spectral index, the fluid and pattern velocity difference, the cases of continuous jet versus discrete component, the relevance of adiabatic losses in the observer's frame, and others.

We can instead use the overall separation velocity

$v_{\text {sep }}=\mu_{\text {sep }} D_{\theta}(1+z)=\frac{2 \beta c \sin \theta}{1-\beta^{2} \cos ^{2} \theta}$,

where $D_{\theta}$ is the angular size distance and $\mu_{\text {sep }}$ is the summed proper motion of jet and counter jet with respect to the core, to obtain an independent relation between $\theta$ and $\beta$. In Fig. 6 we show the regions of the $(\theta, \beta)$ parameter space that are allowed by our measurements. These results indicate a misaligned nature, with a viewing angle in the range $10^{\circ} \leq \theta \leq 21^{\circ}$, and the jet velocity in the range $(0.28 \leq \beta \leq 0.43$ or $1.04 \leq$ Lorentz factor $\leq 1.11$ ). The corresponding values of the Doppler factor decrease from $\delta \sim 1.6$ in the top left part of the selected area to $\delta \sim 1.3$ in the bottom right.

The proposed scenario is not the only viable one. We could relax the assumption of simultaneous ejection of the NW and SE components or invoke different interactions on the two sides as the cause of the observed asymmetries. While these scenarios would not allow us to set any meaningful constraints and are therefore less interesting to explore, the unambiguous motion with subluminal velocity in the NW jet and the presence of emission on both sides of the core argue strongly for an overall misaligned and only mildly relativistic jet.

\section{Discussion and conclusion}

Our analysis confirms the detection (TS $>90$ ) of faint $\gamma$-ray emission $\left(L_{\gamma} \sim 6 \times 10^{41} \mathrm{erg} \mathrm{s}^{-1}\right)$ from the radio galaxy NGC 3894 . 


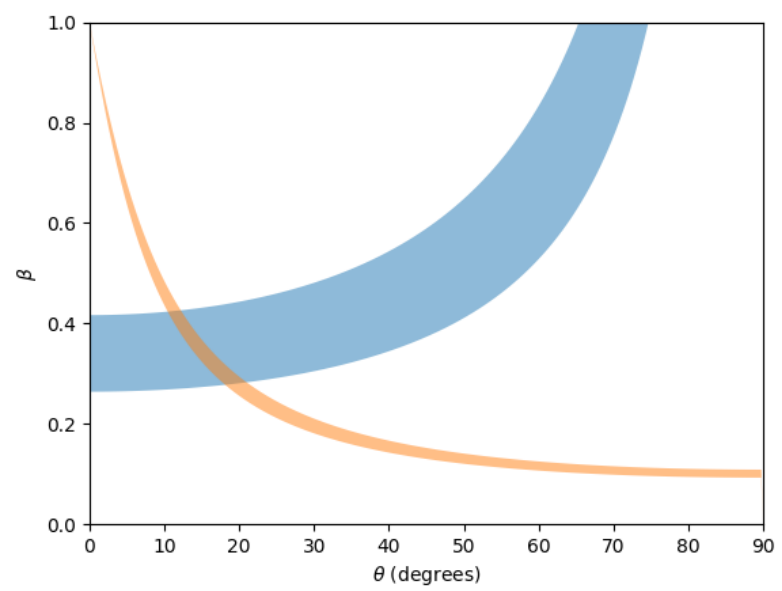

Fig. 6. $(\theta, \beta)$-plane, with constraints from the jet and counter-jet armlength ratio (in blue) and the separation velocity (in orange); the colored regions indicate the $3 \sigma$ confidence ranges.

The radio VLBA analysis based on the extended time baseline yields a somewhat smaller viewing angle $\left(10^{\circ}<\theta<21^{\circ}\right)$ than the previous estimates of Taylor et al. (1998), nevertheless, it is still suggestive of a more misaligned nature in comparison with typical $\gamma$-ray blazars, an overall compact size, and low or moderate bulk motion. The misaligned scenario for NGC 3894 is further supported by a number of multi-wavelength properties reported in the literature. The main results supporting this scenario are the lack of polarization in the radio images (Tremblay et al. 2016), the absence of flaring activity in both radio and $\gamma$-ray data (only slow variability is observed in the decade-long monitoring with the Owens Valley Radio Observatory; Richards et al. 2011) ${ }^{7}$, and the optical spectrum and the low polarization $(0.4-0.5 \%)$, which do not indicate continuum emission other than from stars (Marcha et al. 1996).

Clues to the origin of the $\gamma$-ray emission in this source can come from a comparison with the other classes of extragalactic $\gamma$-ray emitters. Figure 7 shows the diagram of the $\gamma$-ray luminosity versus spectral index for the jetted AGNs with known redshift in the 4LAC. The high-luminosity side of the plot $\left(L_{\gamma} \gtrsim 10^{43} \mathrm{erg} \mathrm{s}^{-1}\right)$ is populated by blazar sources, classified as flat-spectrum radio quasars (FSRQs), BL Lac sources (BLLs), and steep-spectrum radio quasars (SSRQs). Their high luminosities and the frequently observed extreme variability favor an origin of the emission in a compact, highly relativistic region, likely within the aligned inner (subkpc) jet (Urry \& Padovani 1995; Padovani et al. 2017). Conversely, radio galaxies (RDGs) are characterized by lower levels of luminosities and variability (with a few notable exceptions), and it is an open question whether this is a consequence of the larger jet inclination angles or of an inherently different site or mechanism for the $\gamma$-ray emission with respect to blazars.

The $\gamma$-ray luminosity of NGC 3894 is several orders of magnitude lower than that of CSSs, the other intrinsically compact radio objects. Although the CSSs reported in the 4LAC are much farther away $(z>0.6)$, they are all quasars, and their high luminosity suggests that relativistic boosting is likely to play a role in their $\mathrm{GeV}$ detection.

NGC 3894 is one of the lowest luminosity $\left(L_{\gamma}<10^{42} \mathrm{erg} \mathrm{s}^{-1}\right)$ radio galaxies contained in $4 \mathrm{LAC}$, and it appears to share the same $\gamma$-ray properties as its evolved misaligned counterparts.

\footnotetext{
7 See http://www. astro.caltech.edu/ovroblazars/data. php? page $=$ data_return\&source $=\mathrm{J} 1148+5924$
}

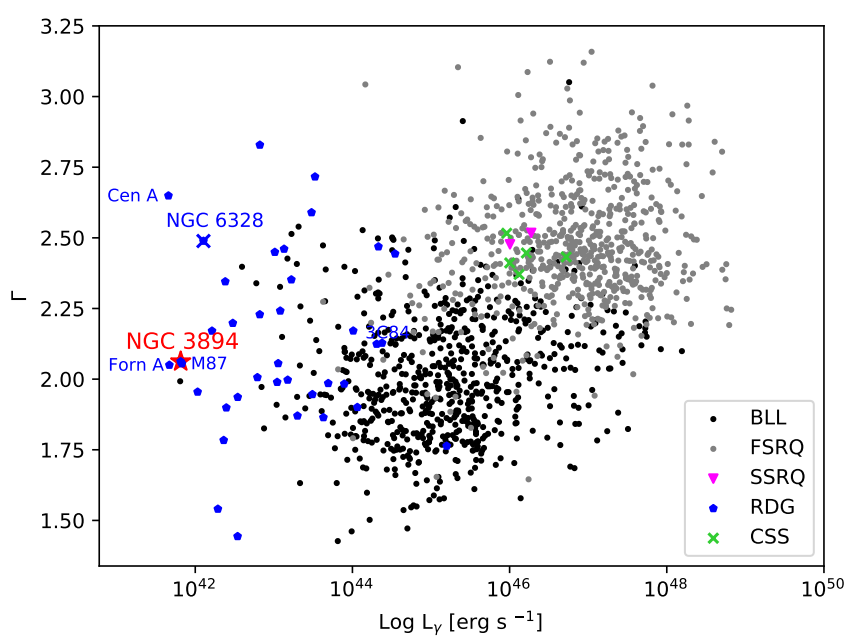

Fig. 7. Diagram of the $\gamma$-ray luminosity vs. spectral index for the extragalactic sources with known redshift contained in the 4LAC. The young radio galaxy NGC 6328 (also called PKS 1718-649), which is the first young radio source detected at $\gamma$-rays, is also labeled in the plot.

Similar luminosities are also observed for the extended radio galaxies Centaurus A and M 87. However, these two are less distant $(z<0.006)$, as is the other bona fide young radio source detected by Fermi-LAT, NGC 6328 (Migliori et al. 2016).

The location of the $\gamma$-ray emitting region in radio galaxies is still controversial, with either the jet or the lobes being plausible emitting sites. At small scales, a clear link between the ejection of bright superluminal knots from the radio core and intense $\gamma$-ray flaring has been observed in the radio galaxies 3C 111 and 3C 120 (Grandi et al. 2012; Casadio et al. 2015; Tanaka et al. 2015). On the other hand, at large distances from the nucleus, a firm detection of $\mathrm{GeV}$ emission from the extended radio lobes of Cen A and Fornax A has been reported in Abdo et al. (2010) and Ackermann et al. (2016).

The most natural object among the misaligned sources for a comparison is the other CSO detected by LAT, NGC 6328 (Migliori et al. 2016). The two sources have similar (low) $L_{\gamma}$, linear sizes and dynamically estimated ages. The inner structure of NGC 3894 is as small as $\sim 5 \mathrm{pc}$ and its dynamic age is $\sim 60$ years, whereas the estimated linear size and dynamic age of NGC 6328 are $\sim 2 \mathrm{pc}$ and $\sim 100 \mathrm{yr}$ (Migliori et al. 2016). This seems to suggest that the detection of CSOs is limited to the closest targets and could be connected to the very first evolutionary stages. However, NGC 3894 has a flatter photon index $(\Gamma=2.01 \pm 0.10)$ than NGC $6328(\Gamma \sim 2.49 \pm 0.18)$. Interestingly, this could be related to their different radio morphologies: NGC 6328 shows mini lobes and no clear detection of jets, while NGC 3894 exhibits a jet (more collimated) structure. It might therefore be speculated that in the former CSO, the $\gamma$-ray emission could come from the compact lobes, as proposed by some models (Stawarz et al. 2008), whereas it originates from the jet.

Two additional interesting sources for a comparison are the radio galaxies M $87(z=0.0044)$ and 3 C 84 (also called NGC $1275, z=0.0176)$. Both sources have a viewing angle similar to NGC 3894: $15^{\circ}<\theta<25^{\circ}$ (Mertens et al. 2016) and $\theta<18^{\circ}$ (Giovannini et al. 2018), respectively. The velocities of the jet components are about $0.2 c$ in 3C 84 (Suzuki et al. 2012) and in the inner part of M 87 (Mertens et al. 2016), similar to what we find for NGC 3894. The three sources have a comparable photon index $(\Gamma \sim 2.1)$. Although it is less distant and more extended $(\sim 100 \mathrm{kpc})$, M 87 shows a $\gamma$-ray luminosity of $6-7 \times 10^{41} \mathrm{erg} \mathrm{s}^{-1}$, similar to NGC 3894. Different is the case 
of 3C 84, which has two-sided compact (parsec scale) jets and a distance similar to NGC 3894, but it presents a $\gamma$-ray luminosity more than 200 times higher $\left(2 \times 10^{44} \mathrm{erg} \mathrm{s}^{-1}\right)$. Unlike NGC 3894, for which no observations with Cherenkov telescopes have been performed, M 87 and 3C 84 are also detected at very high energy (VHE, $E>100 \mathrm{GeV}$; Aharonian et al. 2006; Aleksić et al. 2012). In particular, at VHE, M 87 displayed strong variability on timescales as short as one day, but no unique signature of the region responsible for the VHE flares has been identified (Abramowski et al. 2012). Despite the long-term monitoring of M 87 during 2012-2015 in a low-activity state, the production site of $\gamma$-rays remains unclear (MAGIC Collaboration 2020). However, the correlation observed between the radio and X-ray activities is a strong indication that most often the emitting region is close to the core in this source. In the case of $3 \mathrm{C} 84$, several works on radio, X-ray, and $\gamma$-ray variability suggest that short-term and long-term variability may be produced in different regions of the source. In particular, short-term variability seems related to the injection of fresh particles that are accelerated in a shock in the core region, whereas long-term variability is more likely connected with the jet structure (e.g., Fukazawa et al. 2016; Hodgson et al. 2018). Similarly to M 87 and 3C 84, long-term radio and X-ray monitoring of NGC 3894 may provide important information about the origin of the $\gamma$-ray emission.

When we consider the $\gamma$-ray properties of NGC 3894 together with M 87, Cen A, NGC 6328, and Fornax A, we explor the high-energy emission from a mildly relativistic region $\left(\Gamma_{\text {bulk }} \leq 1.1\right)$ and sample the low-power tail of the jet activity. The case of the two CSOs NGC 3894 and NGC 6328 is particularly interesting: beyond being misaligned and low-power radio sources, they represent the first two cases of young ( $\lesssim 100$ years) radio sources detected by Fermi-LAT. Comparing these two CSO with two more evolved radio galaxies, Cen A and M 87, which have a similar luminosity $L_{\gamma}$ and photon index $\Gamma$, we were able to note a possible connection between the photon index and the viewing angle. While Cen A (Tingay et al. 1998) and NGC 6328 have a large viewing angle $\theta>50^{\circ}$ and a steep spectrum $\Gamma>2.5$, NGC 3894 and M 87 present a small viewing angle $\theta<25^{\circ}$ and a flat spectrum $\Gamma \sim 2$.

Variable emission observed in the same time interval in radio and $\gamma$-rays can be useful to localize the origin of the high-energy emission in AGN. However, no significant variability has been observed in $\gamma$-rays for NGC 3894 (see also Fig. 3). The lack of variability in $\gamma$-rays is expected for young radio sources with the high-energy emission produced in the lobes. At radio frequencies, a long-term and dense radio monitoring performed with the Owens Valley Radio Observatory between 2008 and 2019 shows only slow variability of the total flux (Richards et al. 2011). For a deep study of the radio emission and morphology variability at milliarcsec scale, we lack observations in the period between 2007 and 2017. New VLBA radio observations, together with an extended multiwavelength analysis, including radio, optical, $\mathrm{X}$-ray and $\gamma$-ray data, will be the subject of future work. In particular, further radio analysis at milliarcsec scale will be essential to continue monitoring the jet expansion and the variability of their components in order to investigate the origin of the $\gamma$-ray emission in this source. Radio observations at arcsec scale may provide important information on the surrounding environments, as well as the connection of the source with largescale emissions. As previously discussed, X-ray data play a key role in distinguishing between emission mechanisms (accretion or ejection) and in verifying the properties of the environment. Finally, further studies of the $\gamma$-ray emission will be fundamental for investigating the origin of its high-energy emission.
Acknowledgements. The Fermi-LAT Collaboration acknowledges generous ongoing support from a number of agencies and institutes that have supported both the development and the operation of the LAT as well as scientific data analysis. These include the National Aeronautics and Space Administration and the Department of Energy in the United States, the Commissariat à l'Energie Atomique and the Centre National de la Recherche Scientifique/Institut National de Physique Nucléaire et de Physique des Particules in France, the Agenzia Spaziale Italiana and the Istituto Nazionale di Fisica Nucleare in Italy, the Ministry of Education, Culture, Sports, Science and Technology (MEXT), High Energy Accelerator Research Organization (KEK) and Japan Aerospace Exploration Agency (JAXA) in Japan, and the K. A. Wallenberg Foundation, the Swedish Research Council and the Swedish National Space Board in Sweden. Additional support for science analysis during the operations phase is gratefully acknowledged from the Istituto Nazionale di Astrofisica in Italy and the Centre National d'Etudes Spatiales in France. This work performed in part under DOE Contract DE-AC02-76SF00515. Portions of this research performed at the Naval Research Laboratory (T.J.J., C.C.C.) were supported by NASA DPR S-15633-Y. This research made use of the Astrogeo VLBI FITS image database.

\section{References}

Abdo, A. A., Ackermann, M., Ajello, M., et al. 2009, Phys. Rev. D, 80, 122004 Abdo, A. A., Ackermann, M., Ajello, M., et al. 2010, Science, 328, 725 Abramowski, A., Acero, F., Aharonian, F., et al. 2012, ApJ, 746, 151 Ackermann, M., Ajello, M., Baldini, L., et al. 2016, ApJ, 826, 1

Aharonian, F., Akhperjanian, A. G., Bazer-Bachi, A. R., et al. 2006, Science, 314,1424

Aleksić, J., Alvarez, E. A., Antonelli, L. A., et al. 2012, A\&A, 539, L2

Atwood, W. B., Abdo, A. A., Ackermann, M., et al. 2009, ApJ, 697, 1071

Atwood, W., Albert, A., Baldini, L., et al. 2013, 2012 Fermi Symp. Proc. - eConf C121028 [arXiv:1303.3514]

Bruel, P., Burnett, T. H., Digel, S. W., et al. 2018, 8th International Fermi Symp. [arXiv:1810.11394]

Casadio, C., Gómez, J. L., Grandi, P., et al. 2015, ApJ, 808, 162

D’Ammando, F., Orienti, M., Giroletti, M., \& Fermi Large Area Telescope Collaboration 2016, Astron. Nachr., 337, 59

de Vaucouleurs, G., de Vaucouleurs, A., Corwin, Jr., H. G., et al. 1991, Third Reference Catalogue of Bright Galaxies (New York, NY (USA): Springer)

Fukazawa, Y., Shiki, K., Tanaka, Y., Itoh, R., \& Nagai, H. 2016, ApJ, submitted [arXiv:1608.03652]

Giovannini, G., Savolainen, T., Orienti, M., et al. 2018, Nat. Astron., 2, 472 Gonçalves, A. C., \& Serote Roos, M. 2004, A\&A, 413, 97

Grandi, P., Torresi, E., \& Stanghellini, C. 2012, ApJ, 751, L3

Greisen, E. W. 2003, Astrophys. Space Sci. Lib., 285, 109

Hodgson, J. A., Rani, B., Lee, S.-S., et al. 2018, MNRAS, 475, 368

Kino, M., \& Asano, K. 2011, MNRAS, 412, L20

MAGIC Collaboration (Acciari, V. A., et al.) 2020, MNRAS, 492, 5354

Marcha, M. J. M., Browne, I. W. A., Impey, C. D., \& Smith, P. S. 1996, MNRAS, 281, 425

Massaro, F., Thompson, D. J., \& Ferrara, E. C. 2015, A\&ARv, 24, 2

Mattox, J. R., Bertsch, D. L., Chiang, J., et al. 1996, ApJ, 461, 396

McConville, W., Ostorero, L., Moderski, R., et al. 2011, ApJ, 738, 148

Mertens, F., Lobanov, A. P., Walker, R. C., \& Hardee, P. E. 2016, A\&A, 595,

A54

Migliori, G., Siemiginowska, A., Sobolewska, M., et al. 2016, ApJ, 821, L31

Müller, C., Kadler, M., Ojha, R., et al. 2014, A\&A, 562, A4

Padovani, P., Alexander, D. M., Assef, R. J., et al. 2017, A\&ARv, 25, 2

Peck, A. B., \& Taylor, G. B. 1998, ApJ, 502, L23

Peck, A. B., \& Taylor, G. B. 2000, ApJ, 534, 90

Perlman, E. S., Stocke, J. T., Conway, J., \& Reynolds, C. 2001, AJ, 122, 536

Petrov, L., Gordon, D., Gipson, J., et al. 2009, J. Geod., 83, 859

Principe, G., Malyshev, D., Ballet, J., \& Funk, S. 2018, A\&A, 618, A22

Readhead, A. C. S., Taylor, G. B., Xu, W., et al. 1996, ApJ, 460, 612

Richards, J. L., Max-Moerbeck, W., Pavlidou, V., et al. 2011, ApJS, 194, 29

Sahakyan, N., Baghmanyan, V., \& Zargaryan, D. 2018, A\&A, 614, A6

Shepherd, M. C., Pearson, T. J., \& Taylor, G. B. 1994, BAAS, 26, 987

Stawarz, Ł., Ostorero, L., Begelman, M. C., et al. 2008, ApJ, 680, 911

Suzuki, K., Nagai, H., Kino, M., et al. 2012, ApJ, 746, 140

Tanaka, Y. T., Doi, A., Inoue, Y., et al. 2015, ApJ, 799, L18

Taylor, G. B., Wrobel, J. M., \& Vermeulen, R. C. 1998, ApJ, 498, 619

The Fermi-LAT Collaboration 2019, ArXiv e-prints [arXiv:1905. 10771]

The Fermi-LAT Collaboration 2020, ApJS, 247, 33

Tingay, S. J., Jauncey, D. L., Reynolds, J. E., et al. 1998, AJ, 115, 960

Tremblay, S. E., Taylor, G. B., Ortiz, A. A., et al. 2016, MNRAS, 459, 820

Urry, C. M., \& Padovani, P. 1995, PASP, 107, 803

Wood, M., Caputo, R., Charles, E., et al. 2017, Proc. 35th ICRC, Busan, South

Korea, PoS(ICRC2017), 824

Wrobel, J. M., Jones, D. L., \& Shaffer, D. B. 1985, ApJ, 289, 598 